\title{
Knowing Me, Knowing You: Anal Gland Secretion of European Badgers (Meles meles) Codes for Individuality, Sex and Social Group Membership
}

\author{
Michael J. Noonan ${ }^{1,2}$ - Helga V. Tinnesand ${ }^{3}$ - Carsten T. Müller ${ }^{4}$ - Frank Rosell ${ }^{3}$. David W. Macdonald ${ }^{5}$. \\ Christina D. Buesching ${ }^{5}$
}

Received: 5 July 2019 / Revised: 30 August 2019 / Accepted: 3 October 2019/Published online: 7 November 2019

(C) The Author(s) 2019

\begin{abstract}
European badgers, Meles meles, are group-living in the UK, and demarcate their ranges with shared latrines. As carnivores, badgers possess paired anal glands, but olfactory information on the content of badger anal gland secretion (AGS) is largely uninvestigated. Here, we examined the volatile organic compounds (VOCs) of AGS samples from 57 free-living badgers using solid-phase microextraction (SPME) and gas chromatography-mass spectrometry. AGS was rich in alkanes (C7-C15, 14.3\% of identified compounds), aldehydes (C5-C14, 9.7\%), phenols (C6-C15, 9.5\%), alcohols (C5-C10, 7.3\%), aromatic hydrocarbons (C6-C13, 6.8\%), ketones (C6-C13, 6.3\%) and carboxylic acids (C3-C12, 5.6\%) and contained a variety of esters, sulfurous and nitrogenous compounds, and ethers. The number of VOCs per profile ranged from 20 to 111 (mean $=65.4 ; \pm 22.7 S D$ ), but no compound was unique for any of the biological categories. After normalization of the raw data using Probabilistic Quotient Normalization, we produced a resemblance matrix by calculating the Euclidian distances between all sample pairs. PERMANOVA revealed that AGS composition differs between social groups, and concentration and complexity in terms of number of measurable VOCs varies between seasons and years. AGS VOC profiles encode individual identity, sex and vary with female reproductive state, indicating an important function in intraspecific communication. Because AGS is excreted together with fecal deposits, we conclude that chemical complexity of AGS enables particularly latrine-using species, such as badgers, to advertise more complex individual-specific information than in feces alone.
\end{abstract}

Keywords Chemical communication $\cdot$ Latrine use $\cdot$ Olfaction $\cdot$ Fitness advertisement $\cdot$ Volatile organic compounds

\section{Introduction}

The environmental persistence of olfactory cues ensures their efficiency in indirect communication, where the

Michael J. Noonan, Helga V. Tinnesand and Christina D. Buesching are co-first authors.

Electronic supplementary material The online version of this article (https://doi.org/10.1007/s10886-019-01113-0) contains supplementary material, which is available to authorized users.

Christina D. Buesching

christina.buesching@zoo.ox.ac.uk

1 National Zoological Park, Smithsonian Conservation Biology Institute, 1500 Remount Rd, Front Royal, VA 22630, USA

2 Department of Biology, University of Maryland, College Park, MD 20742, USA signaler is not required to encounter other individuals directly to convey information (Johnston 2008). Thus, many mammals use their feces as a means of intra-specific communication in the context of individual advertisement,
3 Faculty of Technology, Natural Sciences and Maritime Sciences, Department of Natural Sciences and Environmental Health, University College of Southeast Norway, 3800 Bø, Telemark, Norway

4 School of Biosciences, Cardiff University, Sir Martin Evans Building, Museum Avenue, Cardiff CF10 3AX, UK

5 Wildlife Conservation Research Unit, Department of Zoology, Recanati-Kaplan Centre, University of Oxford, Tubney House, Abingdon Road, Tubney, Abingdon, Oxfordshire OX13 5QL, UK 
reproduction, and territoriality (Brown and Macdonald 1985). Especially in carnivores, where encounters can lead to mortality, feces play an important role in conspecific communication (Gorman and Trowbridge 1989). Many species deposit their feces at specific latrine sites, which can be used by several individuals from the same or neighboring social groups (reviewed in Buesching and Jordan 2019). Feces may carry information about family- or social group-membership (Carthey et al. 2018) because gut flora composition can reflect an individual's social contact network (Tung et al. 2015). In addition, because the liver/gut axis is the predominant excretory pathway for steroid hormone metabolites in many mammals (Umapathy et al. 2013), faeces can also encode information on reproductive status (Martín et al. 2010), stress levels (Schatz and Palme 2001), and/or dominance status (Creel 2001). Nevertheless, fecal odor is heavily affected by diet (Ferkin et al. 1997) and digestive processes (Shirasu and Touhara 2011), and therefore fecal deposits alone are typically limited in their suitability as differentiated, individual-specific advertisement signals.

In carnivores, anal gland secretion (AGS) is produced by paired anal glands, sac-like structures located in the rectum either side of the anus (Macdonald 1985). The AGS is excreted together with fecal deposits through mechanical stimulation during defecation (McColl 1967). Additionally, some species are able to voluntarily evert, e.g. spotted hyaena Crocuta crocuta: (Burgener et al. 2009) or empty their anal glands through muscular contraction e.g. skunks Mephitis sp., Conepatus sp., Spilogale sp. (Blackman 1911). Most mustelid AGS are rich in organosulfur compounds and carboxylic acids (reviewed in Buesching and Stankowich 2017), and in many species the chemical profile of AGS has been shown to be individual-specific, e.g. Eurasian otter, Lutra lutra (Kean et al. 2011), steppe polecat, Mustela eversmanii (Zhang et al. 2002), Siberian weasel, Mustela sibirica (Zhang et al. 2002), and ferret, Mustela furo (Zhang et al. 2005). The AGS also often encodes additional information about age, e.g. giant panda Ailuropoda melanoleuca (Yuan et al. 2004); sex, e.g. Eurasian otter (Kean et al. 2011) and ferret (Zhang et al. 2005); reproductive status, e.g. Eurasian otter (Kean et al. 2011); and group membership, e.g. spotted hyaenas (Theis et al. 2012). The information content of fecal odors ise thus heavily moderated by AGS (Macdonald 1985), which enables the conveyance of more complex, individual-specific information than feces alone (Buesching and Jordan 2019; Buesching and Stankowich 2017). This capacity for individual and/or group-level advertisement is particularly important in species that defecate in latrines used by several individuals/ social groups (e.g. Delahay et al. 2000) in order to make possible unambiguous matching of fecal odor information available through excreted metabolic byproducts to the marking individual.
European badgers, Meles meles, are particularly tractable models for investigating the socio-ecological importance of AGS encoded information. Badgers rely primarily upon olfaction to gain information about their environment and conspecifics (Buesching and Macdonald 2001). Consequently, olfactory cues play a central role in structuring their socio-spatial organization (Buesching and Macdonald 2001; Delahay et al. 2000; Tinnesand et al. 2015). Badgers establish composite latrines containing feces, urine, and AGS, the location of which can remain stable over decades (Delahay et al. 2000). Over time, badger latrines can thus accumulate upwards of several hundred individual fecal deposits (Delahay et al. 2000). In scent-provisioning experiments wild badgers have been found to react differently to AGS from individuals in reproductive condition versus non-reproductively active animals (Tinnesand et al. 2015). Individuals also proved capable of distinguishing between feces (Palphramand and White 2007) and AGS (Tinnesand et al. 2015) of members from their own group, from those of neighbors and of strangers. These findings suggest that, mechanistically, badger AGS should encode some level of individually identifiable information. In this regard, however, the only previous study investigating the chemical composition of badger AGS found no evidence of AGS containing any information about individuality, sex or reproductive status, although they did find indication of group differences coded in long-chained, non-volatile compounds, thus positing a long-term territorial function (Davies et al. 1988). However, because Davies et al. (1988) used solvent extraction that may lead to an under-representation and/or loss of volatiles, which have been shown to code for individualspecific characteristics in other species (Buesching and Stankowich 2017), and compared only one captive with one free-living group, these results are difficult to generalize. Consequently, a comprehensive understanding of the role of AGS in badger communication is lacking.

Here, we apply to badger AGS an extraction method designed specifically to analyze the Volatile Organic Compounds (VOCs) using solid-phase micro extraction (SPME; Pawliszyn et al. 1997) and gas-chromatographymass spectrometry (GC-MS; Stein 1999). We then test the resulting chemical profiles for differences related to (a) individual identity, (b) sex, (c) reproductive state, (d) group differences, and (e) temporal variation between seasons and years. Guided by the behavioral reactions observed in previous scent provisioning experiments (Tinnesand et al. 2015), we predict badger AGS to contain individual-specific information about the signaler (such as individuality, sex and reproductive state) as well as information pertaining to groupmembership. We expect information pertaining to individuality and group-membership to remain stable, and thus recognizable by conspecifics, over time. 
Table 1 Samples used in chemical analyses of anal gland secretion of European badgers (Meles meles)

\begin{tabular}{llllcc}
\hline Year & $N$ per Year & Season & $N$ per Season & $N$ Males & $N$ Females \\
\hline 2010 & \multirow{2}{*}{59} & Spring & 17 & 4 & 13 \\
& & Summer & 22 & 11 & 11 \\
& & Autumn & 20 & 11 & 9 \\
2011 & \multirow{2}{*}{25} & Spring & 9 & 6 & 3 \\
& & Summer & 10 & 7 & 3 \\
& & Autumn & 6 & 4 & 2 \\
Total & 84 & & 84 & 43 & 41 \\
\hline
\end{tabular}

\section{Methods and Materials}

\section{Study Animals and Sample Collection}

Anal gland secretions were collected from 57 individuals belonging to 19 different social groups from a high-density badger population in Wytham Woods, Oxfordshire, UK (51:46:26 N / 1:19:19 W). Details are given in Table 1 and Table S1 in the Supplementary Material. As part of an ongoing long-term research project, and following the methodology described in Macdonald et al. (2009), badgers were trapped three times annually in 2010 and 2011: (i) in spring (May/ June) after cubs were fully weaned and coinciding with the peak in latrine activity (Delahay et al. 2000), (ii) in summer (August/September) during lowest food abundance and least latrine activity (Kilshaw et al. 2009), and (iii) in autumn (November) during reproductive quiescence (Sugianto et al. 2018) when badgers reach their maximum body weight and latrine use is concentrated at feeding sites (Buesching et al. 2016).

Badgers were trapped overnight in cage traps baited with peanuts, and traps were checked between 6.30-8.00 am. Captured animals were transferred to holding cages and transported to a central field station, before being sedated by an intramuscular injection of $0.2 \mathrm{ml}$ ketamine hydrochloride/ kg body weight ('Ketamidor': Chanelle, Hungerford, Berkshire, U.K: McLaren et al. 2005). At first capture (usually as cubs), all badgers received a permanent unique tattoo in the left inguinal region. Thus, all animals could be identified individually, sexed, and aged. Cubs and yearlings (i.e. all individuals younger than 2 years) were excluded from this study to ensure sexual maturity (Buesching et al. 2009; Sugianto et al. 2019). In males, reproductive status was inferred from the degree of testicular descent from the body cavity into the scrotum and categorized as $0=$ not in reproductive condition (testes not visible and fully retracted into the body cavity, not mobile), $1=$ intermediate (testes slightly ascended and restricted mobility), or $2=$ in reproductive condition: testes fully descended (both testes clearly discernible by visual inspection separated by the scrotal septum and mobile in the scrotal sack:
Buesching et al. 2009. In females, estrus was inferred from vulva condition and categorized as $0=$ anestrous (vulva skincolored, non-swollen/flat and dry); $1=$ intermediate (vulva skin-colored, slightly swollen but dry), or $2=$ estrous (vulva pink, swollen and mucous membranes moist; Buesching et al. 2002; but see Sugianto et al. 2018). The sett of capture was assumed to be the sett of residence (Macdonald et al. 2008), and social group membership was attributed from baitmarking surveys (Delahay et al. 2000).

AGS samples were obtained by gently everting and palpating the left anal gland. The liquid secretion was collected in sterile glass vials with Teflon ${ }^{\circledR}$ lined caps. Contact between badger skin and the inside of the vials was carefully avoided. Samples were frozen immediately, and stored at $-20{ }^{\circ} \mathrm{C}$ prior to chemical analysis.

\section{Sample Preparation and Chemical Analyses}

Chemical analyses were performed during one week in February and one week in June 2012 in Bø, Norway. Each day, 7 to 10 samples were analyzed in randomized order and the equipment was not used for any other purpose during either of these periods. After homogenization by shaking the sample for $30 \mathrm{~s}$ by hand, a standardized amount $(0.1 \mathrm{~g})$ of secretion was transferred into a clean SPME vial (Supelco, Oslo, Norway), and left at room temperature for $30 \mathrm{~min}$ to equilibrate, before being placed into a water bath at $40{ }^{\circ} \mathrm{C}$ to ensure a stable temperature during extraction. SPME fibers (Stableflex divinylbenzene/carboxene/polydimethylsiloxane (DVB/CAR/PDMS) 50/30 $\mu \mathrm{m}$ bonded fiber; Supleco) were exposed to the headspace of each sample for $60 \mathrm{~min}$. Fibers were conditioned according to the manufacturer's recommendations, and reconditioned in the injection port of the GC-MS at $260{ }^{\circ} \mathrm{C}$ for $10 \mathrm{~min}$ after each sample, and again for $30 \mathrm{~min}$ when the fiber had not been used for several hours. After every five samples, a combined system/fiber blank was run, where the fiber was analyzed without being exposed to a sample in order to detect any contamination or fiber deterioration. If deterioration was detected, the fiber was discarded. An additional system/fiber blank was run with a new fiber to ensure that there was no contamination in the GC-MS, and then the previous samples were re-analyzed in reverse order until the GC-MS results obtained with the new fiber matched those obtained with the old fiber.

Fibers were analyzed immediately after sample exposure. A HP6890 Series II gas chromatograph (Agilent, Oslo, Norway) equipped with a non-polar HP-5 MS column (5\% phenyl-methyl-siloxane; $30.0 \mathrm{~m}$ long $\times 0.25 \mathrm{~mm}$ ID $\times$ $0.25 \mu \mathrm{m}$ film thickness; Agilent) and connected to a HP 5973 Series mass spectrometer detector (Agilent) was used. The split/splitless inlet was used in the splitless mode with helium as carrier gas at a constant flow of $0.9 \mathrm{~mL} \mathrm{~min}^{-1}$. Fibers were inserted manually and desorbed for two minutes 
at $260^{\circ} \mathrm{C}$ in the injection port fitted with a Merlin Microseal ${ }^{\circledR}$ (SigmaAldrich, Oslo, Norway) and SPME liner (Supelco). The oven was held at $40{ }^{\circ} \mathrm{C}$ for two minutes, then increased by $1.5^{\circ} \mathrm{C} / \mathrm{min}$ to $100{ }^{\circ} \mathrm{C}, 4{ }^{\circ} \mathrm{C} / \mathrm{min}$ to $150{ }^{\circ} \mathrm{C}$ and finally $10{ }^{\circ} \mathrm{C} / \mathrm{min}$ to $270{ }^{\circ} \mathrm{C}$ (hold $15 \mathrm{~min}$ ). The temperatures of the transfer line, ion source, and analyzer of the MS was set to $310{ }^{\circ} \mathrm{C}, 230{ }^{\circ} \mathrm{C}$ and $150{ }^{\circ} \mathrm{C}$, respectively. Mass spectra were recorded without solvent delay from $\mathrm{m} / \mathrm{z} 30$ to 550 . A standard mixture of unbranched alkanes between $\mathrm{C} 8$ and $\mathrm{C} 40$ (SigmaAldrich) was injected using an auto-injection system (Agilent 7683 Series Injector) after every 10 samples to calculate Retention Indices (RI) allowing the standardization of retention times and tentative identification of compounds.

Before each use, all laboratory equipment was cleaned in ethanol and acetone and then placed in a high temperature oven at $400{ }^{\circ} \mathrm{C}$ for two hours, in order to remove any contamination with organic compounds. To prevent unstable conditions and to correct for possible changes in the instrument's sensitivity during analyses, the MS was mass-calibrated and auto-tuned each morning.

\section{Data Processing and Tentative Compound Identification}

Raw data were deconvoluted and integrated using the Automated Mass Spectral Deconvolution and Identification System (AMDIS V2.72) against a retention indexed custom mass spectral library. Only compounds that showed a match factor larger or equal to $80 \%$ within the NIST library search (National Institute of Standards and Technology (NIST) Mass Spectral Library V2.0; 2014) and a better than 10\% match in retention index were included and classed as tentatively identified. Where the NIST library did not provide a good match, the compound was given an ID based on its RI match and added as 'unidentified' to the search library in AMDIS, allowing the compound to be identified in other samples. As the main goal of this study was to compare odour profiles between different categories, we did not identify compounds any further.

\section{Statistical Analyses}

Because GC-MS profiles carry relative, and not absolute, information, even small differences in sample volume, concentration, in combination with impurities and background noise, can influence the apparent abundance of compounds, i.e., the 'size effect' (Noonan et al. 2018). Because of this size effect, pre-processing must be carried out before profiles can be compared statistically. We used Probabilistic Quotient Normalization (PQN; Dieterle et al. 2006), which controls for the size effect by calibrating all profiles against the median profile (i.e., median peak values over all samples). Unlike other normalization methods, statistical analyses on PQN transformed data tend to have low false-positive rates, and can accurately recover groups of interest without introducing artefactual differences (Noonan et al. 2018).

To evaluate the complexity of samples (measured as the number of VOCs) across groups of interest (i.e., year, season, sex, reproductive status, and social group), we used two-tailed permutation tests, as described by Strasser and Weber (1999), and implemented in the R package "coin" (Hothorn et al. 2008). We then calculated the Euclidean distance between the normalized profiles of all pairs of samples to produce a resemblance matrix, and performed a permutational multivariate analysis of variance (PERMANOVA; Anderson 2001) test, using the R package "vegan" (Oksanen et al. 2013), to determine if there were any significant differences in AGS profiles from different groups of interest (i.e., year, season, individuals, sex, reproductive status, and social group membership). After testing for significance, we used a random forest (RF) model (Ho 1995) to classify individual AGS profiles according to class and identify the key biomarkers, with PQN normalized peak values as the prediction variables. These analyses were conducted using the $\mathrm{R}$ package "randomForest" (RColorBrewer and Liaw 2018). We chose $\mathrm{RF}$ modeling as it does not require any parameter reduction prior to analysis (Cutler et al. 2007), and has been shown to provide reliable results for biomarker identification (Noonan et al. 2018). Identification of biomarkers important for classifying groups of interest in each RF model was carried out by applying a $k$-means clustering algorithm across $\mathrm{RF}$ variable importance values, and peaks in the cluster with the greatest mean variable importance were classified as a 'peak of primary importance' (PPI; Noonan et al. 2018).

\section{Results}

\section{AGS Profile Composition}

Across all profiles, we found a total of 214 different chemical compounds, of which 209 occurred in more than one sample, and 132 could be tentatively identified (Table 2). No compound was present in all samples, but 2-butyl-2-octenal $(\mathrm{RI}=1378)$, a hexanal dimer, and an unidentified compound $(\mathrm{RI}=1180)$ were present (i.e. measurable) in 82 of the 84 samples. Tentatively identified compounds were primarily alkanes (C7 - C15, 14.3\% of identified compounds), aldehydes (C5 - C14, 9.7\% of identified compounds), phenols (C6 - C15, 9.5\% of identified compounds), alcohols (C5 $\mathrm{C} 10,7.3 \%$ of identified compounds), aromatic hydrocarbons (C6 - C13, 6.8\% of identified compounds), ketones (C6 $\mathrm{C} 13,6.3 \%$ of identified compounds) and carboxylic acids (C3 - $\mathrm{C} 12,5.6 \%$ of identified compounds). AGS samples also contained a considerable number of esters, sulfurous and nitrogenous compounds, and ethers. The chemical complexity 
Table 2 Tentatively identified compounds in the anal gland secretion of Eurasian badgers, Meles meles, with Retention Times (RT min), functional group, and Retention Index (RI medians \pm median absolute deviation). $N$ indicates in how many profiles of the 84 analysed AGS samples the respective compound was found

\begin{tabular}{|c|c|c|c|c|}
\hline Tentatively identified compound & RT (min) & Functional group & RI & $N$ \\
\hline Butanal, 3-methyl- & 2.1 & Aldehyde & $652 \pm 5(214)$ & 46 \\
\hline Benzene & 2.2 & Aromatic hydrocarbon & $654 \pm 11(226)$ & 25 \\
\hline 1-Hexene, 4-methyl- & 2.2 & Hydrocarbon & $659 \pm 1(22)$ & 31 \\
\hline Butanal, 2-methyl & 2.2 & Aldehyde & $662 \pm 8(146)$ & 29 \\
\hline Hexane, 2-methyl- & 2.2 & Hydrocarbon & $667 \pm 1(72)$ & 5 \\
\hline Pentane, 2, 3,- dimethyl & 2.2 & Hydrocarbon & $672 \pm 2(71)$ & 6 \\
\hline Hexane, 3-methyl- & 2.3 & Hydrocarbon & $676 \pm 1(78)$ & 31 \\
\hline 1-Penten-3-ol & 2.4 & Alcohol & $684 \pm 4(58)$ & 21 \\
\hline Pentane, 3-ethyl- & 2.4 & Hydrocarbon & $686 \pm 1(62)$ & 6 \\
\hline Unidentified & 2.4 & Hydrocarbon & & 7 \\
\hline Unidentified & 2.5 & & & 10 \\
\hline Cyclopentane, 1,2-dimethyl-, trans & 2.5 & Ether & $692 \pm 2(34)$ & 10 \\
\hline Unidentified & 2.6 & & & 19 \\
\hline Pentanal & 2.6 & Aldehyde & $699 \pm 5(147)$ & 34 \\
\hline Unidentified & 2.6 & Hydrocarbon & & 4 \\
\hline Unidentified & 2.6 & Hydrocarbon? & & 31 \\
\hline Propanoic acid, ethyl ester & 2.8 & Ester & $709 \pm 4(63)$ & 21 \\
\hline Acetic acid, propyl ester & 2.8 & Ester & $708 \pm 8(55)$ & 7 \\
\hline Propanoic acid & 2.9 & Carboxylic acid & $700 \pm 20(26)$ & 61 \\
\hline Cyclohexane, methyl- & 3.0 & Hydrocarbon & $728 \pm 5(102)$ & 28 \\
\hline Butanoic acid, methyl ester & 3.0 & Ester & $722 \pm 3(59)$ & 4 \\
\hline 2,4-Hexadiene, 2-methyl- & 3.1 & Hydrocarbon & $729 \pm 23(2)$ & 9 \\
\hline Butanenitrile, 3-methyl- & 3.1 & Nitrogen containing & $731 \pm 1(3)$ & 14 \\
\hline Unidentified & 3.1 & & & 16 \\
\hline Cyclopentane, ethyl- & 3.1 & Hydrocarbon & $737 \pm 3(56)$ & 9 \\
\hline 1-Butanol, 3-methyl- & 3.2 & Alcohol & $736 \pm 5(154)$ & 20 \\
\hline 1-Butanol, 2-methyl- & 3.2 & Alcohol & $739 \pm 5(105)$ & 9 \\
\hline Methyl Isobutyl Ketone & 3.3 & Ketone & $735 \pm 5(31)$ & 44 \\
\hline Disulfide, dimethyl & 3.4 & Sulfur containing & $746 \pm 6(126)$ & 20 \\
\hline Pentane, 2,3,4-trimethyl- & 3.4 & Hydrocarbon & $753 \pm 3(51)$ & 5 \\
\hline Unidentified & 3.4 & & & 21 \\
\hline Unidentified & 3.4 & & & 25 \\
\hline 2-Pentanone, 3-methyl- & 3.5 & Ketone & $752 \pm 4(17)$ & 11 \\
\hline Toluene & 3.8 & Aromatic hydrocarbon & $763 \pm 8(328)$ & 62 \\
\hline Propanoic acid, 2-methyl- & 3.8 & Carboxylic acid & $772 \pm 18(38)$ & 49 \\
\hline Unidentified & 3.9 & Hydrocarbons & & 11 \\
\hline Acetic acid, 2-methylpropyl ester & 4.0 & Ester & $771 \pm 6(43)$ & 7 \\
\hline 4-Methyl-1,4-heptadiene & 4.2 & Hydrocarbon & $767 \pm 20(2)$ & 9 \\
\hline 1-Octene & 4.4 & Hydrocarbon & $789 \pm 4(103)$ & 18 \\
\hline 1,4-Cyclohexadiene, 1-methyl- & 4.7 & Hydrocarbon & $790 \pm 4(5)$ & 14 \\
\hline Hexanal & 4.7 & Aldehyde & $800 \pm 2(453)$ & 47 \\
\hline Butanoic acid, ethyl ester & 4.8 & Ester & $802 \pm 2(154)$ & 8 \\
\hline Butanoic acid & 4.9 & Carboxylic acid & $805 \pm 17(102)$ & 34 \\
\hline 2-Octene, (E)- & 4.9 & Hydrocarbon & $798 \pm 1(30)$ & 23 \\
\hline 2-Octene, $(\mathrm{Z})$ - & 4.9 & Hydrocarbon & $804 \pm 3(27)$ & 9 \\
\hline Unidentified & 5.1 & & & 37 \\
\hline Unidentified & 5.2 & Hydrocarbon? & & 11 \\
\hline
\end{tabular}


Table 2 (continued)

\begin{tabular}{|c|c|c|c|c|}
\hline Tentatively identified compound & $\mathrm{RT}(\min )$ & Functional group & RI & $N$ \\
\hline Unidentified & 5.2 & Hydrocarbon & & 7 \\
\hline Unidentified & 5.2 & Hydrocarbon & & 16 \\
\hline 1,3-Octadiene & 5.6 & Hydrocarbon & $827 \pm 1(9)$ & 4 \\
\hline 2-Pentanone, 3-ethyl- & 6.0 & Ketone & $838 \pm \mathrm{N} / \mathrm{A}(1)$ & 23 \\
\hline Cyclohexane, 1,3,5-trimethyl- & 6.4 & Hydrocarbon & $853 \pm 3(7)$ & 4 \\
\hline Unidentified & 6.7 & & & 70 \\
\hline Ethylbenzene & 6.9 & Aromatic hydrocarbon & $855 \pm 10(202)$ & 25 \\
\hline Butanoic acid, 2-methyl- & 7.1 & Carboxylic acids & $861 \pm 14(91)$ & 61 \\
\hline Benzene, 1,4-dimethyl- & 7.2 & Aromatic hydrocarbon & $865 \pm 7(178)$ & 34 \\
\hline Butanoic acid, 3-methyl- & 7.4 & Carboxylic acids & $863 \pm 16(120)$ & 63 \\
\hline 3-Heptanone & 8.2 & Ketone & $887 \pm 3(33)$ & 8 \\
\hline Benzene, 1,2-dimethyl- & 8.3 & Aromatic hydrocarbon & $887 \pm 9(182)$ & 11 \\
\hline 2-n-Butyl furan & 8.4 & Ether & $893 \pm 1(17)$ & 11 \\
\hline 2-Heptanone & 8.4 & Ketone & $891 \pm 2(212)$ & 6 \\
\hline Nonane & 8.8 & Hydrocarbon & 900 & 13 \\
\hline Unidentified & 8.9 & & & 10 \\
\hline Heptanal & 8.9 & Aldehyde & $901 \pm 2(292)$ & 13 \\
\hline Pentanoic acid & 9.7 & Carboxylic acids & $903 \pm 17(48)$ & 25 \\
\hline$\alpha$ - Thujene (bicyclo[3.1.0]hex-2-ene, 2-methyl-5-(1-methylethyl) & 10.3 & Hydrocarbon & $929 \pm 2(489)$ & 25 \\
\hline$\alpha$-Pinene (2,6,6-Trimethylbicyclo[3.1.1]hept-2-ene) & 10.5 & Hydrocarbon & $929 \pm 7(7)$ & 75 \\
\hline Camphene (bicyclo[2.2.1]heptane, 2,2-dimethyl-3-methylene-) & 11.4 & Hydrocarbon & $952 \pm 2(610)$ & 36 \\
\hline 2(5H)-Furanone, 5,5-dimethyl- & 12.0 & Ester & $952 \pm 5(10)$ & 8 \\
\hline 3-Heptanone, 6-methyl- & 12.1 & Ketone & $941 \pm \mathrm{N} / \mathrm{A}(1)$ & 19 \\
\hline Benzaldehyde & 12.2 & Aldehyde & $962 \pm 3(416)$ & 43 \\
\hline 4-Octen-3-ol, 2,2-dimethyl- & 12.2 & Alcohol & NA & 8 \\
\hline Dimethyl trisulfide & 12.6 & Sulfur containing & $970 \pm 7(154)$ & 10 \\
\hline Sabinene (bicyclo[3.1.0]hexane, 4-methylene-1-(1-methylethyl)-) & 13.2 & Hydrocarbon & $974 \pm 2(619)$ & 71 \\
\hline 1-Octen-3-one & 13.7 & Ketone & $979 \pm 2(176)$ & 17 \\
\hline 1-Octen-3-ol & 13.9 & Alcohol & $980 \pm 2(355)$ & 20 \\
\hline Phenol & 14.3 & Phenols & $980 \pm 4(94)$ & 74 \\
\hline Furan, 2-pentyl- & 14.6 & Ether & $993 \pm 2(178)$ & 17 \\
\hline Myrcene (1,6-octadiene, 7-methyl-3-methylene) & 14.6 & Hydrocarbon & $991 \pm 2(841)$ & 25 \\
\hline 2-Hexenal, 2-ethyl- & 15.2 & Aldehyde & $999 \pm 30(6)$ & 10 \\
\hline Hexanoic acid & 15.6 & Carboxylic acid & $990 \pm 16(133)$ & 4 \\
\hline 3-Carene (bicyclo[4.1.0]hept-3-ene, 3,7,7-trimethyl) & 15.7 & Hydrocarbon & $1011 \pm 2(336)$ & 42 \\
\hline$\alpha$-Terpinene (1,3-Cyclohexadiene, 1-methyl-4-(1-methylethyl)-) & 16.3 & Hydrocarbon & $1017 \pm 2(534)$ & 15 \\
\hline$o$-Cymene (benzene, 1-methyl-2-(1-methylethyl)-) & 16.8 & Aromatic hydrocarbon & $1022 \pm 2(116)$ & 16 \\
\hline Unidentified & 16.8 & & & 8 \\
\hline Unidentified & 17.1 & & & 77 \\
\hline 2,3,4,5-Tetramethylcyclopent-2-en-1-ol & 17.3 & Alcohol & NA & 22 \\
\hline Cyclohexanone, 2,2,6-trimethyl- & 17.4 & Ketone & $1036 \pm 5(14)$ & 12 \\
\hline Unidentified & 18.4 & Carboxylic acid?? & & 12 \\
\hline Unidentified & 19.0 & & & 11 \\
\hline Unidentified & 19.0 & & & 36 \\
\hline$\gamma$-Terpinene (1,4-Cyclohexadiene, 1-methyl-4-(1-methylethyl)-) & 19.0 & Hydrocarbon & $1060 \pm 3(739)$ & 21 \\
\hline 2-Octenal, (E)- & 19.2 & Aldehyde & $1060 \pm 3(124)$ & 7 \\
\hline 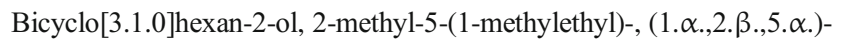 & 19.5 & Alcohol & $1070 \pm 4(50)$ & 3 \\
\hline Unidentified & 20.1 & & & 37 \\
\hline 5-Nonanone & 20.1 & Ketone & $1073 \pm 4(11)$ & 36 \\
\hline
\end{tabular}


Table 2 (continued)

\begin{tabular}{|c|c|c|c|c|}
\hline Tentatively identified compound & $\mathrm{RT}(\min )$ & Functional group & RI & $N$ \\
\hline Unidentified & 20.4 & & & 15 \\
\hline Unidentified & 20.5 & & & 8 \\
\hline$\delta$-Terpinene (cyclohexene, 1-methyl-4-(1-methylethylidene)-) & 20.7 & Hydrocarbon & $1088 \pm 2(607)$ & 15 \\
\hline Phenol, 2-methoxy- & 20.8 & Diverse & $1090 \pm 3(130)$ & 4 \\
\hline Cyclohexane, 2,4-diethenyl-1-methyl- & 20.9 & Hydrocarbon & NA & 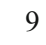 \\
\hline Thujone (bicyclo[3.1.0]hexan-3-one, 4-methyl-1-(1-methylethyl)-), [1S-(1 $1 \alpha, 4 \alpha, 5 \alpha)]-$ & 21.2 & Alcohol & $1103 \pm 2(101)$ & 11 \\
\hline Unidentified & 21.4 & & & 24 \\
\hline Undecane & 21.5 & Hydrocarbon & 1100 & 30 \\
\hline trans,trans-2,9-Undecadiene & 21.7 & Hydrocarbon & NA & 24 \\
\hline Phenylethyl alcohol & 22.0 & Alcohol & $1116 \pm 5(261)$ & 65 \\
\hline 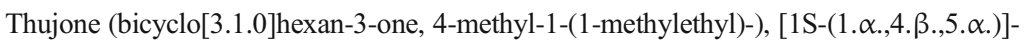 & 22.1 & Ketone & $1114 \pm 2(129)$ & 16 \\
\hline Unidentified & 22.4 & & & \\
\hline Benzyl methyl ketone & 22.7 & Ketone & $1110 \pm 12(5)$ & 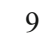 \\
\hline 2-Nonen-4-one & 22.8 & Ketone & $1124 \pm 3(2)$ & 25 \\
\hline Hexanoic acid, 2-ethyl- & 23.0 & Carboxylic acid & $1124 \pm 5(24)$ & 54 \\
\hline Unidentified & 23.6 & & & 43 \\
\hline Bicyclo[3.1.0]hexan-2-one, 5-(1-methylethyl)- & 24.1 & Ketone & $1156 \pm 2(34)$ & 11 \\
\hline Unidentified & 24.2 & & & 22 \\
\hline 3-Cyclohexen-1-ol, 4-methyl-1-(1-methylethyl)-(R)- & 25.0 & Alcohol & $1177 \pm 2(764)$ & 16 \\
\hline (3E,5Z)-1,3,5-Undecatriene & 25.0 & Hydrocarbon & $1174 \pm 1(9)$ & \\
\hline Unidentified & 25.2 & & & 80 \\
\hline 1-Undecene, 2-methyl- & 25.5 & Hydrocarbon & $1185 \pm \mathrm{N} / \mathrm{A}(1)$ & 7 \\
\hline Myrtenal (bicyclo[3.1.1]hept-2-ene-2-carboxaldehyde, 6,6-dimethyl-) & 25.7 & Aldehyde & $1193 \pm 3(145)$ & 59 \\
\hline Phenol, 2,3-dichloro- & 26.0 & Diverse & $1200 \pm 0(3)$ & 10 \\
\hline Dodecane & 26.1 & Hydrocarbon & 1200 & 30 \\
\hline Unidentified & 26.2 & Carboxylic acid?? & & 33 \\
\hline Unidentified & 26.4 & & & 80 \\
\hline 1-Cyclohexene-1-carboxaldehyde, 2,6,6-trimethyl- & 26.7 & Aldehyde & $1220 \pm 3(75)$ & 65 \\
\hline Unidentified & 26.8 & & & 12 \\
\hline Unidentified & 26.8 & & & 7 \\
\hline 3-Ethyl-2-nonanone & 26.9 & Ketone & NA & 60 \\
\hline Unidentified & 27.0 & & & 7 \\
\hline Quinoline & 27.1 & Nitrogen containing & $1237 \pm 5(17)$ & 27 \\
\hline 2-Decenal, (Z)- & 27.2 & Aldehyde & $1252 \pm 2(23)$ & 10 \\
\hline Unidentified & 27.4 & & & 25 \\
\hline Unidentified & 27.5 & & & 76 \\
\hline Unidentified & 27.7 & & & 55 \\
\hline Benzeneacetic acid & 27.8 & Carboxylic acid & $1262 \pm 5(45)$ & 8 \\
\hline Unidentified & 27.8 & & & 12 \\
\hline Acetic acid, 2-phenylethyl ester & 27.9 & Ester & $1258 \pm 3(76)$ & 50 \\
\hline Methyl 8-methyl-nonanoate & 28.2 & Ester & $1277 \pm \mathrm{N} / \mathrm{A}(1)$ & 13 \\
\hline 5-Undecanone & 28.3 & Ketone & NA & 26 \\
\hline Unidentified & 28.3 & & & 29 \\
\hline Unidentified & 28.4 & & & 29 \\
\hline Unidentified & 28.5 & & & 29 \\
\hline Unidentified & 28.6 & & & 25 \\
\hline 3-Undecanone & 28.7 & Ketone & $1283 \pm 8(4)$ & 18 \\
\hline Indole & 28.7 & Nitrogen containing & $1295 \pm 7(85)$ & 37 \\
\hline 2-Undecanone & 28.8 & Ketone & $1294 \pm 2(159)$ & 12 \\
\hline
\end{tabular}


Table 2 (continued)

\begin{tabular}{|c|c|c|c|c|}
\hline Tentatively identified compound & $\mathrm{RT}(\min )$ & Functional group & RI & $N$ \\
\hline Unidentified & 28.9 & & & 26 \\
\hline Ethanone, 1-(2-aminophenyl)- & 28.9 & Diverse & $1308 \pm 8(21)$ & 29 \\
\hline Tridecane & 28.9 & Hydrocarbon & 1300 & 20 \\
\hline Quinoline, 2-methyl- & 29.0 & Nitrogen-containing & $1311 \pm 2(5)$ & 53 \\
\hline Unidentified & 29.0 & & & 9 \\
\hline Unidentified & 29.0 & Carboxylic acid? & & 20 \\
\hline Unidentified & 29.1 & Carboxylic acid? & & 52 \\
\hline Heptanoic acid, 2-ethyl- & 29.3 & Carboxylic acid & NA & 43 \\
\hline Unidentified & 29.4 & & & 7 \\
\hline Unidentified & 29.4 & Carboxylic acid? & & 52 \\
\hline Unidentified & 29.6 & & & 49 \\
\hline Unidentified & 29.8 & & & 32 \\
\hline Unidentified & 29.9 & & & 15 \\
\hline Unidentified & 29.9 & Carboxylic acid? & & 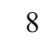 \\
\hline Naphthalene, 1,2-dihydro-1,1,6-trimethyl- & 30.0 & Aromatic Hydrocarbon & $1354 \pm 8(16)$ & 22 \\
\hline Propanoic acid, 2-phenylethyl ester & 30.0 & Diverse & $1350 \pm 1(10)$ & 7 \\
\hline Unidentified & 30.0 & & & 5 \\
\hline 1H-Indene, 2,3-dihydro-1,1,4,6-tetramethyl- & 30.0 & Aromatic Hydrocarbon & $1350 \pm \mathrm{N} / \mathrm{A}(1)$ & 63 \\
\hline 2(3H)-Furanone, dihydro-5-pentyl- & 30.2 & Ester & $1363 \pm 5(81)$ & 26 \\
\hline Tridecane, 2-methyl- & 30.2 & Hydrocarbon & $1364 \pm 1(9)$ & 9 \\
\hline Propanoic acid, 2-methyl-, 3-hydroxy-2,2,4-trimethylpentyl ester & 30.4 & Diverse & $1380 \pm 0(2)$ & \\
\hline 2-Octenal, 2-butyl- & 30.4 & Aldehyde & $1378 \pm 10(9)$ & 81 \\
\hline Unidentified & 30.4 & & & 14 \\
\hline Unidentified & 30.5 & & & 23 \\
\hline Unidentified & 30.6 & Carboxylic acid? & & 52 \\
\hline Propanoic acid, 2-methyl-, 2-phenylethyl ester & 30.7 & Diverse & $1396 \pm 1(4)$ & 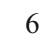 \\
\hline Unidentified & 30.8 & & & 56 \\
\hline Unidentified & 30.9 & & & 80 \\
\hline 2-Undecanone, 6,10-dimethyl- & 30.9 & Ketone & $1408 \pm 2(8)$ & 14 \\
\hline Unidentified & 31.1 & & & 9 \\
\hline Unidentified & 31.2 & & & 67 \\
\hline Naphthalene, 2-butyldecahydro- & 31.3 & Hydrocarbon & $1432 \pm \mathrm{N} / \mathrm{A}(1)$ & 50 \\
\hline Unidentified & 31.4 & & & 17 \\
\hline Unidentified & 31.4 & & & 20 \\
\hline Unidentified & 31.5 & & & 20 \\
\hline 2-Butanone, 4-(2,6,6-trimethyl-1-cyclohexen-1-yl)- & 31.5 & Ketone & $1433 \pm 3(12)$ & 30 \\
\hline Unidentified & 31.5 & & & 33 \\
\hline Unidentified & 31.6 & & & 22 \\
\hline Geranyl acetone (5,9-undecadien-2-one, 6,10-dimethyl-) & 31.7 & Ketone & $1456 \pm 5(14)$ & 19 \\
\hline Hexanedioic acid, bis(1-methylethyl) ester & 31.7 & Ester & $1464 \pm 0(3)$ & 4 \\
\hline Unidentified & 31.8 & & & 10 \\
\hline Unidentified & 31.9 & & & 4 \\
\hline Tetradecane, 3-methyl- & 31.9 & Hydrocarbon & $1470 \pm 1(12)$ & 7 \\
\hline Unidentified & 32.0 & & & 15 \\
\hline 3-Buten-2-one, 4-(2,6,6-trimethyl-1-cyclohexen-1-yl)- & 32.2 & Ketone & $1486 \pm 4(210)$ & 80 \\
\hline Unidentified & 32.2 & & & 13 \\
\hline Butanoic acid, 2-methyl-, 2-phenylethyl ester & 32.2 & Ester & $1488 \pm 3(5)$ & 7 \\
\hline Pentadecane & 32.3 & Hydrocarbon & 1500 & 8 \\
\hline Unidentified & 32.4 & & & 33 \\
\hline
\end{tabular}


Table 2 (continued)

\begin{tabular}{|c|c|c|c|c|}
\hline Tentatively identified compound & $\mathrm{RT}(\min )$ & Functional group & RI & $N$ \\
\hline Unidentified & 32.5 & & & 35 \\
\hline Undecanoic acid, 2-methyl- & 32.6 & Carboxylic acid & $\mathrm{NA}$ & 15 \\
\hline Unidentified & 32.8 & & & 21 \\
\hline 2(4H)-Benzofuranone, 5,6,7,7a-tetrahydro-4,4,7a-trimethyl-, (R)- & 32.8 & Ester & $1532 \pm 10(26)$ & 13 \\
\hline Unidentified & 33.2 & & & 77 \\
\hline 2-Nonenal, 2-pentyl- & 33.3 & Aldehyde & NA & 32 \\
\hline Unidentified & 33.4 & & & 6 \\
\hline Unidentified & 33.7 & & & 80 \\
\hline Unidentified & 33.8 & & & 10 \\
\hline Unidentified & 34.0 & & & 22 \\
\hline Unidentified & 34.2 & & & 25 \\
\hline Unidentified & 34.7 & & & 31 \\
\hline Unidentified & 34.8 & & & 24 \\
\hline
\end{tabular}

of the profiles varied considerably between samples, with the number of VOCs per profile ranging from 20 to 111 (mean = $65.4 \pm 22.7 S D$ ). No compound was confirmed to be unique to any of the biological categories (sex, reproductive status, group-membership) investigated indicating analog rather than digital coding of information.

\section{Temporal Variation in Badger AGS}

A permutation test revealed that, even using the same sampling and laboratory protocols and running samples in a randomized order, AGS samples collected in 2010 contained significantly more VOCs than those collected in 2011 $(P<0.001)$, and the relative structure of profiles differed significantly between years $\left(F_{1,82}=8.48 ; P<0.001\right)$. Interestingly, 56 VOCs were only found in 2010 samples, whereas no compounds were confirmed to be unique to 2011. When clustering based on year, a random forest model classified AGS from 2010 and 2011 with an accuracy of ca. $89.3 \%$ (Fig. 1b). A $k$-means clustering analysis on the variable importance values revealed that inter-annual differences in toluene $(\mathrm{RI}=763)$ and an unidentified fatty acid $(\mathrm{RI}=1311)$ were the PPIs for differentiating between years (Fig. 1a). Consistent with the overall trends, both compounds tended to have higher concentrations in 2010 than in 2011 indicating that all AGS compounds likely occurred in much higher concentrations in 2010 than in 2011, resulting in more VOCs occurring above the peak threshold of our laboratory protocol (Fig. 2).

Although a permutation test revealed no evidence of interseasonal variation in the overall number of VOCs $(P=0.49)$, there was evidence of a substantial difference in AGS profile composition between seasons $\left(F_{2,81}=1.63 ; P=0.018\right)$. A random forest clustering analysis based on peak areas classified seasonal AGS samples with an accuracy of $66.7 \%$ (Fig. 1d). A $k$-means clustering analysis on variable importance values revealed that an unidentified compound $(R I=842)$ was the sole PPI for differentiating between seasons (Fig. 1c). This compound tended to have high values in spring, and decreased throughout the year with values 4 -fold lower by autumn (Fig. 3).

\section{Biological Variation in Badger AGS}

\section{Individuality}

A PERMANOVA on data from individuals with repeat samples over time revealed that inter-individual variation was significantly larger than intra-individual variation $\left(F_{6,14}=1.50\right.$; $P=0.026)$, although there was no evidence of inter-individual variation in the overall number of VOCs $(P=0.62)$. These results indicate that despite the significant seasonal and inter-annual variation described above, differences between individuals were maintained across seasons and years.

\section{Sex}

In line with the inter-individual variation in AGS chemical composition, a permutation test showed no evidence of inter-sexual variation in the overall number of VOCs $(P=$ 0.57). A PERMANOVA however, did evidence significant differences in the relative abundances of compounds between sexes $\left(F_{1,82}=4.57 ; P<0.001\right)$. Random forest clustering based on sex resulted in an accuracy of $86.9 \%$ (Fig. 1f), and a $k$-means clustering analysis on variable importance values revealed that 2-methyl-butanoic acid $(\mathrm{RI}=861), 2$-butyl- 2octenal $(\mathrm{RI}=1378)$, and an unidentified compound $(\mathrm{RI}=$ 1345) were the PPIs for differentiating male and female profiles (Fig. 4). 
Year a)

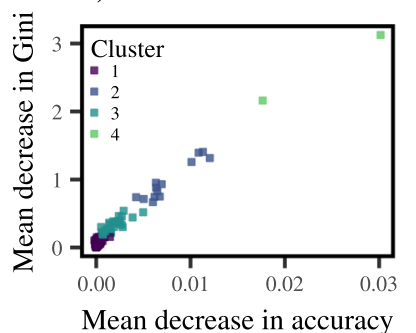

Season

c)

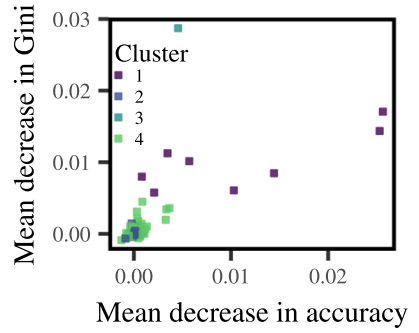

Sex

e)

Group

g)

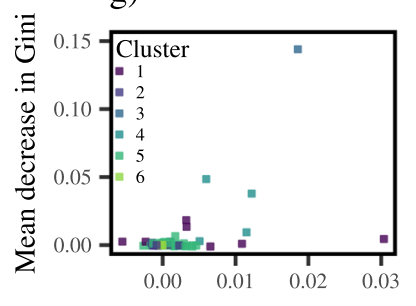

Mean decrease in accuracy

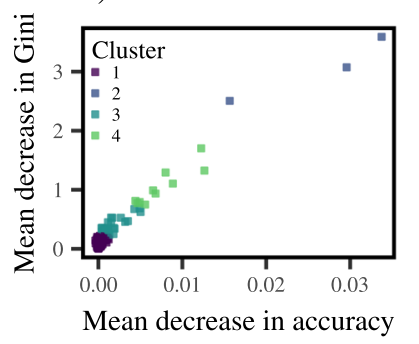

b)

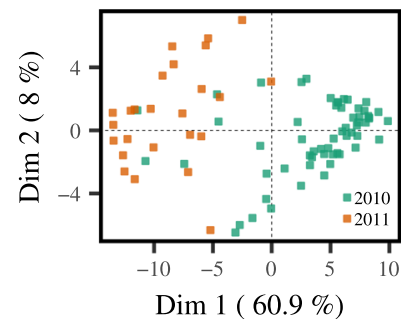

d)

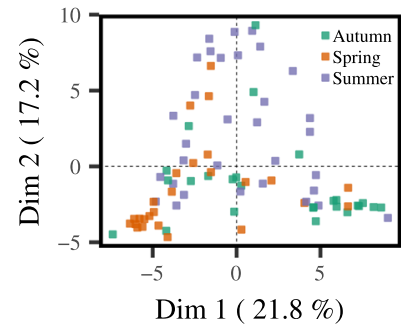

f)

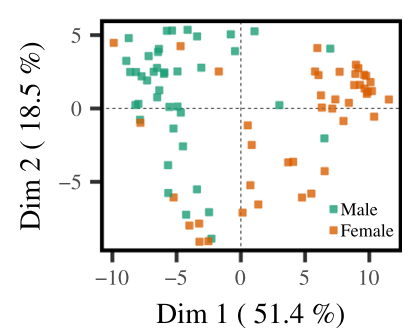

h)

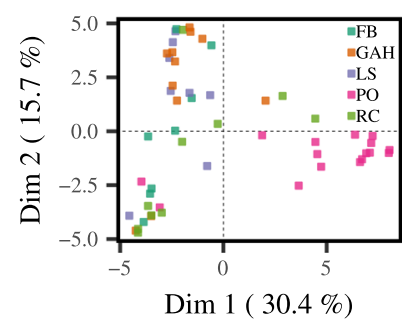

Fig. 1 Scatter plots depicting a $k$-means clustering across variable importance values (a), (c) and (e); and the first two dimensions (Dim) of principal component analyses across the proximity matrices (b), (d) and (f) for random forest models classifying temporal, and biological variation in AGS composition

\section{Reproductive State}

There was no evidence that the overall number of VOCs differed across reproductive states for females $(P=0.37)$ nor males $(P=0.99)$, and we found no evidence that profile structure differed between a)

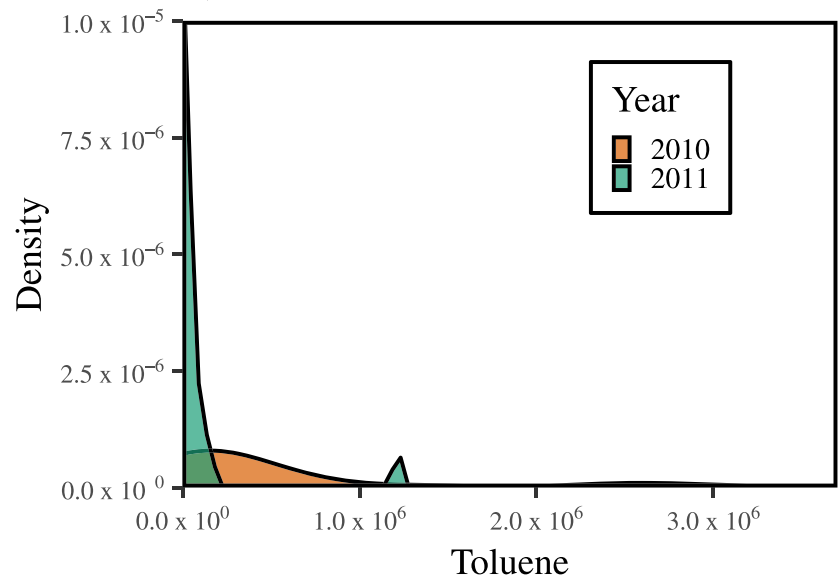

b)

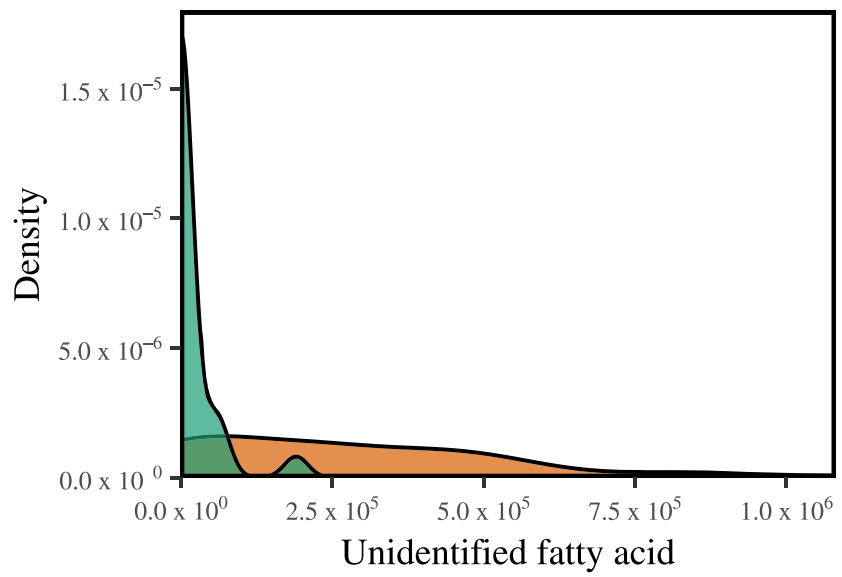

Fig. 2 Density plots of the compounds of primary importance for classifying inter-annual variation in AGS composition

reproductive states for females $\left(F_{2,40}=0.77 ; P=0.70\right)$

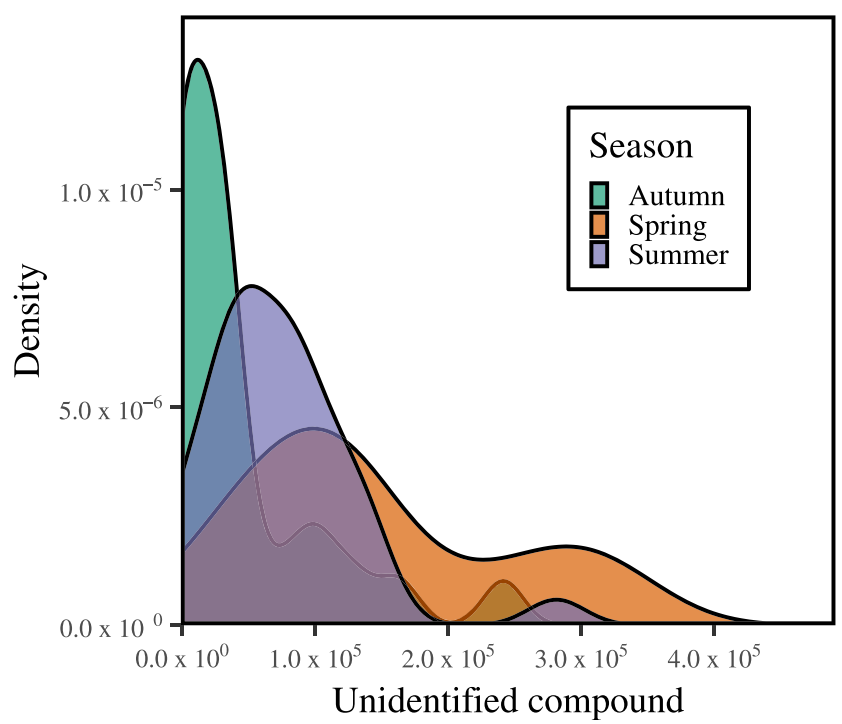

Fig. 3 Density plot of the compound of primary importance for classifying inter-seasonal variation in AGS composition 
a)

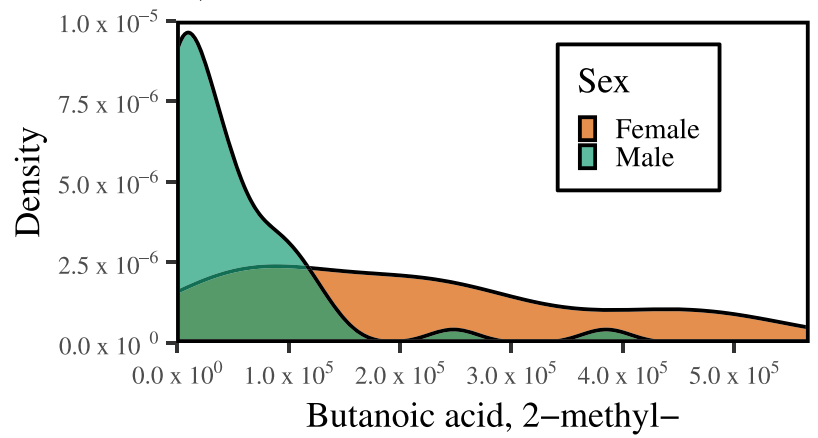

b)

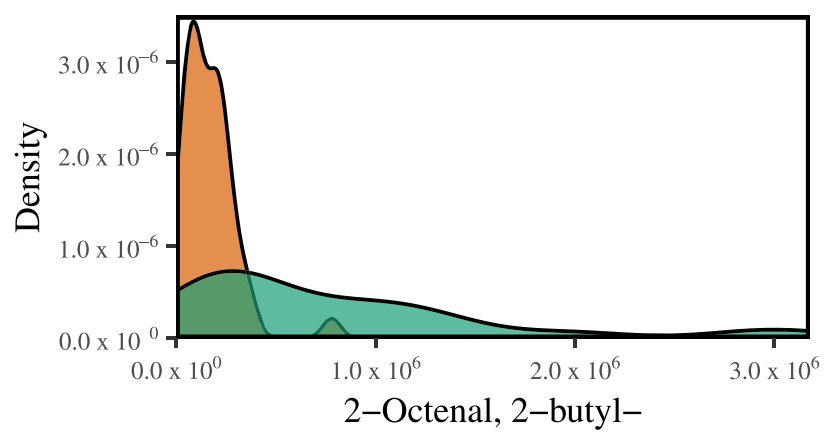

c)

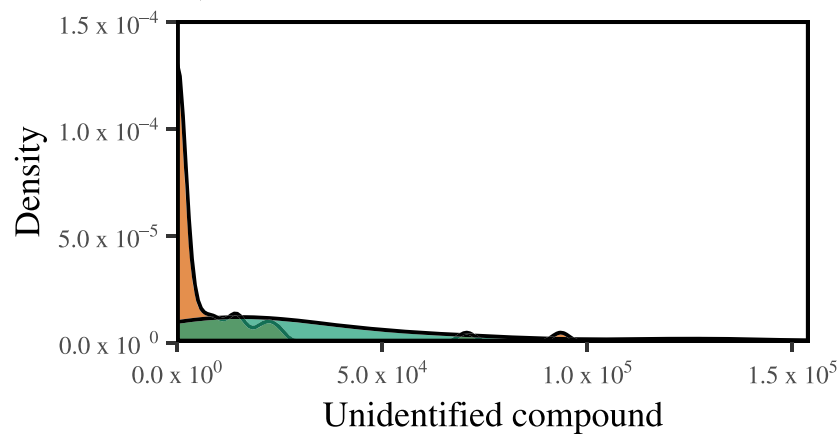

Fig. 4 Density plots of the compounds of primary importance for classifying inter-sexual variation in AGS composition

nor males $\left(F_{2,40}=0.76 ; P=0.77\right)$. Despite the lack of significance, however, random forest clustering based on reproductive state did result in a classification accuracy of $79.4 \%$ for females ( $46.5 \%$ for males; Fig. 5). A $k$-means clustering analysis on variable importance values revealed that 2-methyl-butanoic acid $(\mathrm{RI}=861)$, pentanoic acid $(\mathrm{RI}=903)$, phenol $(\mathrm{RI}=980)$, indole $(\mathrm{RI}=1295)$, and 3 unidentified compounds $(\mathrm{RI}=1256$, 1644 , and 1688 respectively) were the PPIs for differentiating AGS profiles between female reproductive states, whereas for differentiating male reproductive states 2,3,4,5-tetramethylcyclopent-2-en-1-ol, and 2 unidentified compounds ( $\mathrm{RI}=811$, and 842 respectively) were the PPIs.

\section{Group Membership}

There was no evidence of inter-group variation in the overall number of VOCs $(P=0.40)$, but a PERMANOVA revealed significant differences in profiles between groups $\left(F_{4,48}=\right.$ $1.71 ; P<0.005)$. Random forest clustering based on group membership did result in reasonable identifiability, with a model accuracy of $62.6 \%$ (Fig. 1h). A $k$-means clustering analysis on variable importance values revealed that two terpenes $\alpha$-thujene (2-methyl-5-(1-methylethyl)bicyclo[3.1.0]hex-2-ene; RI =929) and sabinene (4-methylene-1-(1-methylethyl)-bicyclo[3.1.0]hexane; RI = 974) were the peaks of primary importance for differentiating between groups. However, permutation tests revealed no significant differences in compound concentrations between groups ( $P=0.116$ and 0.087 respectively).

\section{Discussion}

Although olfactory communication plays a central role in badger socio-spatial organization (Buesching and Macdonald 2001; Delahay et al. 2000; Tinnesand et al. 2015), a mechanistic understanding of how information at latrines is conveyed between individuals has remained a hitherto under-investigated topic. Our results evidence that badger AGS odor profiles are more diverse and encode more individual-specific information than previously concluded (Davies et al. 1988; Gorman et al. 1984). Analyses of AGS VOC's collected by SPME confirmed a large number of VOCs, with a highly individual- and sex-specific composition, which varied with female reproductive state. The AGS VOC's were moderated by groupmembership, and their relative abundance and composition varied between seasons and years.

Carnivores are, by definition, disproportionately fierce and fights between conspecifics may result in severe injuries and/ or death. Thus, many carnivores advertise ownership of essential resources as a pre-emptive measure to avoid conflict (Buesching and Stankowich 2017). This is achieved most efficiently with low-maintenance long-term signals that do not require the continual physical presence of the owner (Buesching and Jordan 2019), but that can be matched to the marking individual (the scent-matching hypothesis Gosling 1986). Inter-group differences in odor profiles were clearly apparent in our badger AGS data, reflecting results from a similar study analyzing the composition of subcaudal gland secretions in this same badger population (Buesching et al. 2002). When combined with the fact that badgers can discern AGS-based information, as evidenced by the variation in response patterns observed in AGS-provisioning experiments (Tinnesand et al. 2015), our findings provide a mechanistic understanding of how AGS serves as a basis for sociological 


\section{Males}

a)

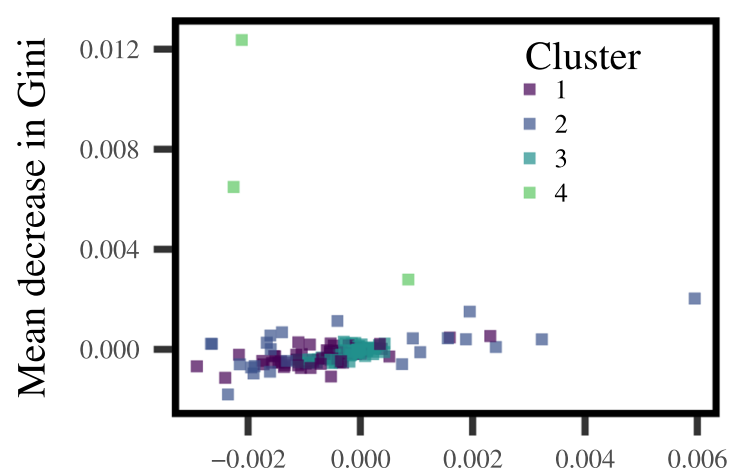

Mean decrease in accuracy b)

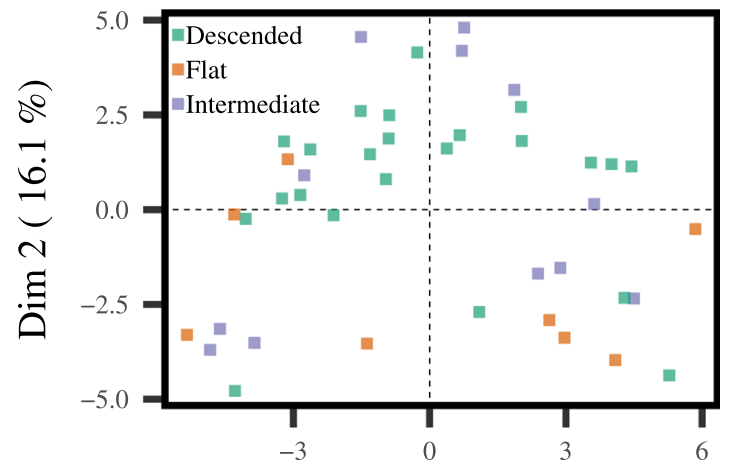

$\operatorname{Dim} 1(23.4 \%)$

\section{Females}

c)

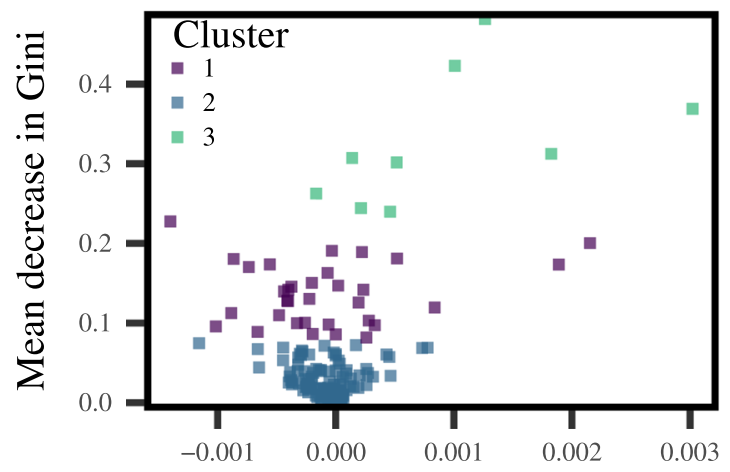

Mean decrease in accuracy

Fig. 5 Scatter plots depicting a k-means clustering across variable importance values (a and c); and the first two dimensions (Dim) of principal component analyses across the proximity matrices (b and $\mathbf{d}$ ) for random

advertisement to conspecifics. Crucially, however, much of the information content in VOCs is temporary. In order to maximize their functionality, territorial scent-marks, which in any non-solitary species need to also include groupmembership information, are expected to be long-lasting in the environment (Buesching and Jordan 2019). Because cue longevity is achieved predominantly by non-volatile compounds, we posit that similar, if not more pronounced, intergroup differences should be apparent in the relative abundance of longer-lived, non-volatile compounds as described in Davies et al. (1988). SPME head space sampling, however, restricted our analyses to volatile compounds only, leaving the question open if badger AGS not only contains informationencoding VOCs (as measured here), but also non-volatile compounds. d)

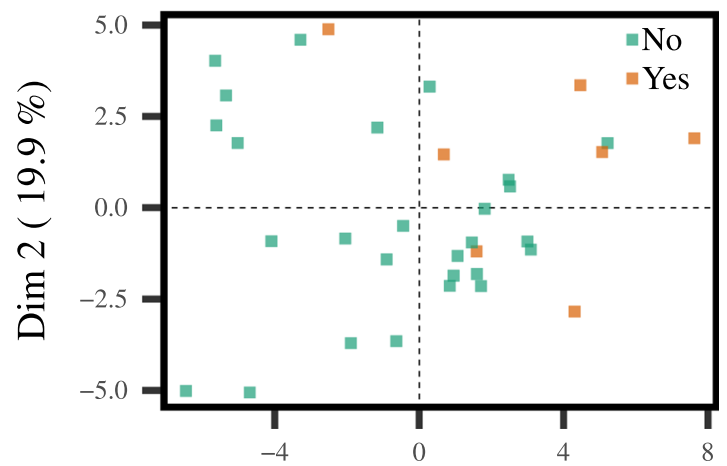

Dim $1(36.6 \%)$

forest models classifying variation in AGS composition across reproductive state for males and females

According to the 'social complexity hypothesis' (reviewed in Freeberg et al. 2012), group-living also places evolutionary pressure on complex communication systems, selecting for increasingly complex individual-specific signals (Pollard and Blumstein 2011). The potential for each individual of a species to have its own olfactory "fingerprint" (Müller-Schwarze 2009) is particularly important in all group-living species that rely on olfactory communication to maintain stable sociospatial networks (Buesching and Jordan 2019). In badgers, because all members of several neighboring social groups use the same latrines, encoding information pertaining to individuality and group-membership in feces and/or AGS is a prerequisite for any individual level advertisement (Buesching and Jordan 2019; Buesching and Macdonald 2001; Buesching and Stankowich 2017), where here we evidence that 
individual AGS profile characteristics remained comparatively stable over time.

Because the ability to find and attract members of the opposite sex as potential mates as well as to identify potential same-sex rivals is crucial for all sexually reproducing species, the ability to encode sex is likely fundamental to advertisement signals (Johnston and DelBarco-Trillo 2009). Unsurprisingly, therefore, sex differences have been confirmed in the AGS from all carnivore species investigated thus far, e.g. banded mongooses, Mungos mungo (Jordan et al. 2011); Eurasian otters (Kean et al. 2011); Brown bears, Ursus arctos (Rosell et al. 2011). In line with these studies, our findings suggest that also in badgers, sex is not encoded digitally through the simple presence/absence of a specific compound in only one of the sexes (Wyatt 2010), but rather in the ratio of compounds (i.e., analogue coding). Although we did not find any statistically significant differences in the chemical composition between different reproductive states, neither in male nor in female AGS profiles, random forest clustering did correctly classify female reproductive state in nearly $80 \%$ of samples. This putative difference is supported by our behavioral observations (Tinnesand et al. 2015), where male badgers exhibited significantly different reactions to AGS from estrous vs non-estrous females. Collectively, these findings evidence that information about female reproductive status is clearly encoded in AGS odor. Crucially, however, in the scent-provisioning experiments of Tinnesand et al. (2015), badgers appeared to lick the AGS samples, indicating the use of their vomero-nasal organ (VNO) to investigate non-volatile compounds, whereas our present study analyzed only VOCs. Indeed, due to its close connection with the reproductive hypothalamus (Sorensen 1996), the VNO is likely to process primarily olfactory information regarding the sex and reproductive status of the sender (Leinders-Zufall et al. 2000). In this regard, we found that the peaks of primary importance for conveying information on reproductive status tended to be less volatile, and have higher RIs than the biomarkers for other forms of information. Interestingly, longer-chained, nonvolatile compounds were also confirmed to correlate with badger reproductive status in their subcaudal gland secretions (Buesching et al. 2002).

Surprisingly, the complexity of AGS odor profiles differed between 2010 and 2011, with the average number of VOCs per profile being significantly higher in 2010 than in the following year. Many of the VOCs found in AGS are probably of dietary origin, i.e. they cannot be produced by mammals, but only by plants, bacteria or invertebrates (Charpentier et al. 2012). Weather variation over this period (Noonan et al. 2014), as well as differences in management of the surrounding farmland, might have resulted in dietary changes causing the observed differences in the chemical composition of AGS. Similarly, Mardon et al. (2010) found that the year of sampling had a significant effect on the chemical profiles of the uropygial secretion of blue petrels, Halobaena caerulea, and related these to differences in climatic conditions, food availability, and factors affecting the birds' metabolism or diet. Contrary to our study, however, Mardon et al. (2010) could not eliminate differences in sample treatment prior to GC-MS analyses as the underlying cause.

In terms of intra-year variation, odor profiles contained the highest numbers of compounds during the spring mating season, but decreased in complexity towards autumn - a period of reproductive quiescence. This mirrors results from GC-MS analyses of badger subcaudal gland secretions (Buesching et al. 2002), as well as visually distinctive differences in chromatograms of the female genital secretion of Coquerel's sifaka, Propithecus coquereli (Greene and Drea 2014). Because scent glands are often under hormonal control (Creel 2001), seasonal variation in secretion composition, e.g. short-beaked echidna, Tachyglossus aculeatus (Harris et al. 2014) and scent-marking activity, e.g. ferret (Chang et al. 2000; red panda, Ailurus fulgens, (Roberts and Kessler 1979), and ringtailed coati, Nasua nasua (Shannon et al. 1995) is typically linked to the species' reproductive cycle. In badgers, patterns in scent-marking activity (Buesching and Macdonald 2004), as well as the chemical characteristics of subcaudal (Buesching et al. 2002) and anal gland secretions (present study), reflect the seasonal sex-steroid patterns of badgers (Buesching et al. 2009; Sugianto et al. 2019) with significantly higher scent-marking activity (Buesching and Macdonald 2004) and latrine usage (Buesching et al. 2016) during the mating and cub-rearing season in late winter/spring.

Badger latrines along borders are used by all members of the adjacent social groups (Buesching et al. 2016; Delahay et al. 2000). While this makes them ideally suited as a node for information exchange between conspecifics (Buesching and Macdonald 2001), it also means encoding individuality is paramount if fecal deposits at group-latrines are to play a role in individual advertisement (Buesching and Macdonald 2001). We provide evidence that VOCs in badger AGS odor profiles can convey a wider range of individual-specific information than was previously assumed through work on longchained, non-volatile compounds (Davies et al. 1988). Because much of the individual-specific information is temporary, e.g. reproductive condition, health- and fitness related information, it is well-suited to be encoded in short-chained highly volatile compounds, which ensure that the signal evaporates (i.e. "expires") in time for individual-specific characteristics to change. VOCs also ensure that scent is discernible from a greater distance. Information specific to group membership in the context of territorial maintenance and defense, in contrast, is unlikely to change over short-to-medium timescales (Buesching and Macdonald 2001), and is thus best encoded in long-lived non-volatile compounds (Davies et al. 1988). Future work will therefore need to analyze and compare odor profiles of both volatile, and non-volatile 
compounds to gain a complete understanding of how individuals encode and perceive information across timescales.

\section{Compliance with Ethical Standards}

Conflict of Interest The authors declare that they have no conflict of interest.

Open Access This article is distributed under the terms of the Creative Commons Attribution 4.0 International License (http:// creativecommons.org/licenses/by/4.0/), which permits unrestricted use, distribution, and reproduction in any medium, provided you give appropriate credit to the original author(s) and the source, provide a link to the Creative Commons license, and indicate if changes were made.

\section{References}

Anderson MJ (2001) A new method for non-parametric multivariate analysis of variance. Austral Ecol 26(1):32-46

Blackman M (1911) The anal glands of mephitus mephitica. Anat Rec 5(11):491-515

Brown RE, Macdonald DW (1985) Social Odours in mammals (volumes 1 \& 2). Oxford University Press, Oxford

Buesching CD, Jordan NR (2019) The social function of latrines: A hypothesis-driven research approach, Springer, chap Chemical Signals in Vertebrates XIV (Ed: C.D. Buesching). Springer International Publishing, Cham, pp 94-106

Buesching CD, Macdonald DW (2001) Scent-Marking Behaviour of the European Badger (Meles meles): Resource Defence or Individual Advertisement? In: Chemical Signals in Vertebrates 9 (Eds: A. Marchlewska-Koj, J. Lepri \& D. Mueller-Schwarze). Springer US, Boston, pp 321-327

Buesching CD, Macdonald DW (2004) Variations in scent-marking behaviour of European badgers Meles meles in the vicinity of their setts. Acta Theriol 49(2):235-246

Buesching C, Stankowich T (2017) Communication amongst the musteloids: signs, signals, and cues, Oxford University Press, chap Biology and conservation of the musteloids (badgers, otters, skunks, raccoons and their kin), pp 149-166

Buesching CD, Waterhouse JS, Macdonald DW (2002) Gaschromatographic analyses of the subcaudal gland secretion of the European badger (Meles meles) part I: chemical differences related to individual parameters. J Chem Ecol 28(1):41-56

Buesching CD, Heistermann M, Macdonald DW (2009) Seasonal and inter-individual variation in testosterone levels in badgers Meles meles: evidence for the existence of two endocrinological phenotypes. J Comp Physiol A 195(9):865-871

Buesching CD, Tinnesand HV, Sin Y, Rosell F, Burke T, Macdonald DW (2016) Coding of Group Odor in the Subcaudal Gland Secretion of the European Badger Meles meles: Chemical Composition and Pouch Microbiota. In: Chemical Signals in Vertebrates 13 (Eds: B.A. Schulte, T.E. Goodwin \& M.H. Ferkin). Springer International Publishing, Cham, pp 45-62

Burgener N, Dehnhard M, Hofer H, East ML (2009) Does anal gland scent signal identity in the spotted hyaena? Anim Behav 77(3):707715

Carthey AJ, Gillings MR, Blumstein DT (2018) The extended genotype: microbially mediated olfactory communication. Trends Ecol Evol

Chang YM, Kelliher KR, Baum MJ (2000) Steroidal modulation of scent investigation and marking behaviors in male and female ferrets (Mustela putorius furo). J Comp Physiol 114(4):401
Charpentier MJ, Barthes N, Proffit M, Bessière JM, Grison C (2012) Critical thinking in the chemical ecology of mammalian communication: roadmap for future studies. Funct Ecol 26(4):769-774

Creel S (2001) Social dominance and stress hormones. Trends Ecol Evol 16(9):491-497

Cutler DR, Edwards TC Jr, Beard KH, Cutler A, Hess KT, Gibson J, Lawler JJ (2007) Random forests for classification in ecology. Ecology 88(11):2783-2792

Davies JM, Lachno DR, Roper TJ (1988) The anal gland secretion of the European badger (Meles meles) and its role in social communication. J Zool 216(3):455-463

Delahay RJ, Brown JA, Mallinson PJ, Spyvee PD, Handoll D, Rogers LM, Cheeseman CL (2000) The use of marked bait in studies of the territorial organization of the European badger (Meles meles). Mammal Rev 30(2):73-87

Dieterle F, Ross A, Schlotterbeck G, Senn H (2006) Probabilistic quotient normalization as robust method to account for dilution of complex biological mixtures. Application in 1H NMR metabonomics. Anal Chem 78(13):4281-4290

Ferkin MH, Sorokin ES, Johnston RE, Lee CJ (1997) Attractiveness of scents varies with protein content of the diet in meadow voles. Anim Behav 53(1):133-141

Freeberg TM, Dunbar RI, Ord TJ (2012) Social complexity as a proximate and ultimate factor in communicative complexity. Philos Trans R Soc Lond Ser B Biol Sci 367(1597):1785-1801

Gorman ML, Trowbridge BJ (1989) The role of odor in the social lives of carnivores. In: Carnivore behavior, ecology, and evolution (Ed: J.L. Gittleman). Springer, pp 57-88

Gorman ML, Kruuk H, Leitch A (1984) Social functions of the subcaudal scent gland secretion of the European badger Meles meles (Carnivora: Mustelidae). J Zool 203(4):549-559

Gosling L (1986) Economic consequences of scent marking in mammalian territoriality. In: Chemical signals in vertebrates 4 (Eds: D. Duvall, D. Mueller-Schwarze \& R.M. Silverstein). Springer, pp 385-395

Greene LK, Drea CM (2014) Love is in the air: sociality and pair bondedness influence sifaka reproductive signalling. Anim Behav $88: 147-156$

Harris R, Holland B, Cameron E, Davies N, Nicol S (2014) Chemical signals in the echidna: differences between seasons, sexes, individuals and gland types. J Zool 293(3):171-180

Ho TK (1995) Random Decision Forests. In: Proceedings of the 3rd International Conference on Document Analysis and Recognition. IEEE Computer Society, Montreal, pp 278-282

Hothorn T, Hornik K, Van De Wiel MA, Zeileis A, et al (2008) Implementing a class of permutation pests: the coin package. Stat Software 28(8)

Johnston RE (2008) Individual odors and social communication: individual recognition, kin recognition, and scent over-marking. Adv Study Behav 38:439-505

Johnston RE, DelBarco-Trillo J (2009) Communication by chemical signals: behavior, social recognition, hormones and the role of the vomeronasal and olfactory systems, Elsevier Academic Press, chap Hormones, Brain and Behavior (Eds: D.W. Pfaff, A.P. Arnold, A.M. Etgen, S.E. Fahrbach \& R.T. Ruben), pp 395-440

Jordan NR, Manser MB, Mwanguhya F, Kyabulima S, Rüedi P, Cant MA (2011) Scent marking in wild banded mongooses: 1. Sex-specific scents and overmarking. Anim Behav 81(1):31-42

Kean EF, Müller CT, Chadwick EA (2011) Otter scent signals age, sex, and reproductive status. Chem Senses 36(6):555-564

Kilshaw K, Newman C, Buesching C, Bunyan J, Macdonald DW (2009) Coordinated latrine use by European badgers, Meles meles: potential consequences for territory defense. J Mammal 90(5):1188-1198

Leinders-Zufall T, Lane AP, Puche AC, Ma W, Novotny MV, Shipley MT, Zufall F (2000) Ultrasensitive pheromone detection by mammalian vomeronasal neurons. Nature 405(6788):792 
Macdonald D (1985) Social odours in mammals, vol 2 (Eds: R.E. Brown \& D.W. Macdonald). Clarendon Press, chap The carnivores: order Carnivora, pp 619-722

Macdonald DW, Newman C, Buesching CD (2008) Male-biased movement in a high-density population of the Eurasian badger (Meles meles). J Zool 89(5):1077-1086

Macdonald DW, Newman C, Nouvellet PM, Buesching CD (2009) An analysis of Eurasian badger (Meles meles) population dynamics: implications for regulatory mechanisms. J Zool 90(6):1392-1403

Mardon J, Saunders SM, Anderson MJ, Couchoux C, Bonadonna F (2010) Species, gender, and identity: cracking petrels' sociochemical code. Chem Senses 35(4):309-321

Martín J, Barja I, López P (2010) Chemical scent constituents in feces of wild Iberian wolves (Canis lupus signatus). Biochem Syst Ecol 38(6):1096-1102

McColl I (1967) The comparative anatomy and pathology of anal glands. Arris and Gale lecture delivered at the Royal College of Surgeons of England on 25th February 1965. Ann R Coll Surg Engl 40(1):36

McLaren GW, Thornton PD, Newman C, Buesching CD, Baker SE, Mathews F, Macdonald DW (2005) The use and assessment of ketamine-medetomidine-butorphanol combinations for field anaesthesia in wild European badgers (Meles meles). Vet Anaesth Analg 32(6):367-372

Müller-Schwarze D (2009) Chemical ecology of vertebrates. Cambridge University Press, Cambridge

Noonan MJ, Markham A, Newman C, Trigoni N, Buesching CD, Ellwood SA, Macdonald DW (2014) Climate and the individual: inter-annual variation in the autumnal activity of the European badger (Meles meles). PLoS One 9(1):e83156

Noonan MJ, Tinnesand HV, Buesching CD (2018) Normalizing gaschromatography-mass spectrometry data: method choice can alter biological inference. BioEssays 40(6):1700210

Oksanen J, Blanchet FG, Kindt R, Legendre P, Minchin PR, O'hara R, Simpson GL, Solymos P, Stevens MHH, Wagner H et al (2013) Package 'vegan'. Community ecology package, version 2(9)

Palphramand KL, White PCL (2007) Badgers, Meles meles, discriminate between neighbour, alien and self scent. Anim Behav 74(3):429 436

Pawliszyn J, Pawliszyn B, Pawliszyn M (1997) Solid phase microextraction (spme). Chem Educ 2(4):1-7

Pollard KA, Blumstein DT (2011) Social group size predicts the evolution of individuality. Curr Biol 21(5):413-417

RColorBrewer S, Liaw MA (2018) Package 'randomforest'University of California, Berkeley: Berkeley, CA, USA

Roberts MS, Kessler DS (1979) Reproduction in red pandas, Ailurus fulgens (Carnivora: Ailuropodidae). J Zool 188(2):235-249

Rosell F, Jojola SM, Ingdal K, Lassen BA, Swenson J, Arnemo JM, Zedrosser A (2011) Brown bears possess anal sacs and secretions may code for sex. J Zool 283(2):143-152

Schatz S, Palme R (2001) Measurement of faecal cortisol metabolites in cats and dogs: a non-invasive method for evaluating adrenocortical function. Vet Res Commun 25(4):271-287
Shannon D, Kitchener A, Macdonald A (1995) The preputial glands of the coati, Nasua nasua. J Zool 236(2):319-322

Shirasu M, Touhara K (2011) The scent of disease: volatile organic compounds of the human body related to disease and disorder. $\mathrm{J}$ Biochem 150(3):257-266

Sorensen P (1996) Biological responsiveness to pheromones provides fundamental and unique insight into olfactory function. Chem Senses 21(2):245-256

Stein SE (1999) An integrated method for spectrum extraction and compound identification from gas chromatography/mass spectrometry data. J Am Soc Mass Spectrom 10(8):770-781

Strasser H, Weber C (1999) On the asymptotic theory of permutation statistics. Math Methods Statist 8(2):220-225

Sugianto NA, Buesching CD, Heistermann M, Newman C, Macdonald DW (2018) Linking plasma sex steroid hormone levels to the condition of external genitalia in European badgers (Meles meles): a critical evaluation of traditional field methodology. Mamm Biol 93:97-108

Sugianto NA, Newman C, Macdonald DW, Buesching CD (2019) Heterochrony of puberty in the European badger (Meles meles) can be explained by growth rate and group-size: evidence for two endocrinological phenotypes. PLoS One 14(3):e0203910

Theis KR, Schmidt TM, Holekamp KE (2012) Evidence for a bacterial mechanism for group-specific social odors among hyenas. Sci Rep 2(1):500

Tinnesand HV, Buesching CD, Noonan MJ, Newman C, Zedrosser A, Rosell F, Macdonald DW (2015) Will trespassers be prosecuted or assessed according to their merits? A Consilient interpretation of territoriality in a group-living carnivore, the European badger (Meles meles). PLoS One 10(7):e0132432

Tung J, Barreiro LB, Burns MB, Grenier JC, Lynch J, Grieneisen LE, Altmann J, Alberts SC, Blekhman R, Archie EA (2015) Social networks predict gut microbiome composition in wild baboons. eLife 4:e05224

Umapathy G, Kumar V, Kabra M, Shivaji S et al (2013) Detection of pregnancy and fertility status in big cats using an enzyme immunoassay based on $5 \alpha$-pregnan-3 $\alpha$-ol-20-one. Gen Comp Endocrinol $180: 33-38$

Wyatt TD (2010) Pheromones and signature mixtures: defining specieswide signals and variable cues for identity in both invertebrates and vertebrates. J Comp Physiol A 196(10):685-700

Yuan H, Liu D, Sun L, Wei R, Zhang G, Sun R (2004) Anogenital gland secretions code for sex and age in the giant panda, Ailuropoda melanoleuca. Can J Zool 82(10):1596-1604

Zhang JX, Sun L, Zhang ZB, Wang ZW, Chen Y, Wang R (2002) Volatile compounds in anal gland of Siberian weasels (Mustela sibirica) and steppe polecats (M. eversmanni). J Chem Ecol 28(6):1287-1297

Zhang J, Soini H, Bruce K, Wiesler D, Woodley S, Baum M, Novotny M (2005) Putative chemosignals of the ferret (Mustela furo) associated with individual and gender recognition. Chem Senses 30(9):727737 